\title{
Outcomes of use of electromagnetic guidance with responsive insertion technology (RIT) during colonoscopy: a prospective randomized controlled trial
}

\section{다(1)우우}

\author{
Authors \\ Shajan Peter ${ }^{1}$, Nipun B. Reddy ${ }^{1}$, Mohammed Naseemuddin ${ }^{1}$, Jenine N. Zaibaq ${ }^{1}$, Gerald McGwin ${ }^{2}$, C. Mel Wilcox ${ }^{1}$
}

Institutions

1 Gastroenterology and Hepatology, University of Alabama at Birmingham, Birmingham, Alabama, United States

2 Epidemiology, University of Alabama at Birmingham, Birmingham, Alabama, United States

submitted 22.3.2018

accepted after revision 5.6.2018

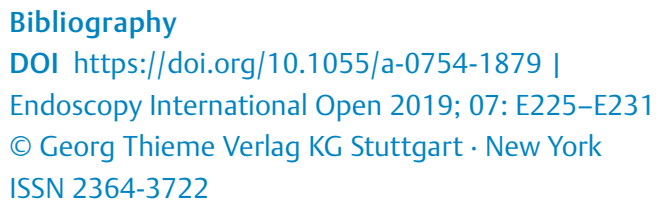

Corresponding author

Shajan Peter, MD, Division of Gastroenterology and Hepatology,, Department of Medicine, University of Birmingham at Alabama, Birmingham, AL 35294-0007, USA Fax: +1-205-975-6201

ssugandha@uabmc.edu

\section{ABSTRACT}

Background and study aims Colonoscopy can be technically challenging and cause discomfort in patients. The integrated Scope Guide assist is built in to show that with its use outcomes are improved during colonoscopy. We aimed to test the usefulness of the Magnetic Scope Guide Assist (ScopeGuide) with respect to cecal intubation time, and other procedural quality outcomes.
Patients and methods We conducted a prospective study of outpatients undergoing elective colonoscopy at the endoscopic units of the University of Alabama at Birmingham (UAB) from March 2016 to July 2016. Patients were randomly assigned in a 1:1 block design to groups that either had standard colonoscopy or Scope-guided colonoscopy. The primary outcome measure was cecal intubation time (CIT). Secondary outcome measures included use of manual pressure, position changes for cecal intubation and sedation requirements.

Results Three hundred patients were randomized to either group; standard $(n=150)$ vs. Scope-guided $(n=150)$. The mean CIT was not statistically different for the standard and the Scope-guided groups (4.6 vs. 4.3 minutes; $P=$ $0.46)$. There were also no statistical differences in frequency of manual pressure applied (16.7\% for Scope-guided vs. $19.1 \%$ for standard; $P=0.65$ ) or position changes $(11.4 \%$ for scope guided vs. $8.8 \%$ standard; $P=0.56)$. Sedation requirements showed lesser use of midazolam $(3.9 \mathrm{mg}$ vs. $4.7 \mathrm{mg}$, $P=0.003)$ in the Scope-guide group, while there was no significant difference in use of fentanyl (fentanyl $-62.1 \mathrm{mg}$ vs. $68.9 \mathrm{mg}, P=0.09$ similar between groups, for Scopeguided vs. standard groups, respectively). Adverse events were similar in both groups.

Conclusions In patients undergoing routine elective colonoscopy, use of ScopeGuide by experienced colonoscopists did not improve CIT or affect the frequency of ancillary maneuvers. The benefit of this device during training of endoscopists could be considered for further studies.

Clinical.Trials.gov

NCT02739893

TRIAL REGISTRATION: single-center, randomized prospective study at clinicaltrials.gov

\section{Introduction}

Colonoscopy is established as the gold standard for diagnosis and management of colonic disorders and for colorectal cancer screening. Given this fact, there is an increasing global demand for use of colonoscopies for both diagnosis and screening re- quired at academic as well as community practice centers nationwide [1]. Colonoscopy can be technically challenging and cause discomfort in patients. The success of performance depends on several factors, such as adequate bowel preparation, anatomy of the patient including previous surgical history, adequate sedation and technical expertise of the endoscopist. 
Accuracy and thoroughness must be balanced with patient safety and comfort. Patient discomfort is typically caused by looping of the colonoscope and improper positioning, thus further resulting in unnecessary stretching of the intestinal lumen.

The integrated ScopeGuide technology offers real-time 3D representation of the shape and position of the endoscope while performing colonoscopy electromagnetically, and was thus designed with the intention of improving procedural efficiency and increasing patient comfort. Frequent colonoscope looping and performance of abdominal maneuvers illustrate the potential need for such a positioning device. In the literature, ScopeGuide has demonstrated some usefulness for training gastroenterology fellows and guiding experienced endoscopists through difficult cases with colonoscopy completion rates and number of attempts at straightening the colonoscope [2]. Another study identified a similar decrease in number of attempts of colonoscope straightening when patients were placed in control of their sedation via patient-controlled analgesia, however, there was no statistically significant difference in analgesia use or patient-reported pain scores between assisted versus unassisted cases performed by an experienced endoscopist [3]. A recent systematic review and meta-analysis of randomized controlled trials studying use of magnetic endoscopic imaging (MEI) as an adjuvant to elective colonoscopy concluded that MEI was associated with a lower failure risk and shorter time to cecal intubation compared to conventional colonoscopy. However, this study was limited as there was a discrepancy in the definition of measurement of cecal intubation time as well as variable definitions of colonoscopist experience, inclusion of relatively small number of studies, and unblinded designs [4]. While these between-study discrepancies and heterogeneity could have led to uncertainty in the meta-analysis estimates, the analysis was well represented and unbiased. We also aimed to study outcomes using the upgraded version of the colonoscope (190 series) which has the incorporated responsive insertion technology (RIT). We therefore aimed to investigate the usefulness of ScopeGuide in assisting colonoscopy with respect to insertion time and other procedural outcomes by experienced endoscopists at a large tertiary referral center.

\section{Patients and methods}

We conducted a randomized study of outpatients undergoing elective colonoscopy performed by trained gastroenterologists at the endoscopic units of the University of Alabama at Birmingham (UAB) from March 2016 to July 2016. Patients were randomly assigned in a 1:1 block design to either Standard colonoscopy or ScopeGuide-assisted colonoscopy. Inclusion criteria included consecutive healthy subjects undergoing colonoscopy at the ambulatory facility of UAB who were ASA (American Society of anesthesiologists) class I-III and had completed their bowel preparation. Exclusion criteria included age <19 years; inability to safely undergo colonoscopy for any reason; coagulopathy (INR > 1.6, prothrombin time $>18 \mathrm{sec}$, thrombocytopenia <80,000 cells $/ \mathrm{mL}$ ); non-English-speaking; history of previous colorectal surgery, suspected severe colonic stric- ture/obstructing tumor, known or suspected bowel obstruction; severe congestive heart failure; disturbances of electrolytes; pregnancy or lactation; or hemodynamic instability. The research study was approved by the UAB Institutional review board (\# F141223002), and all participants provided written informed consent. The study was registered before initiation (Clinical Trials gov. number NCT02739893,). All authors had access to the study data and reviewed and approved the final manuscript.

The adjuvant ScopeGuide ${ }^{\circledR}$ technology works on the principle of embedded magnetic coils throughout the scope with a receiver dish picking up the signal from every single point [3]. These are picked up by a receiver dish located by the side of the patient and sent to the ScopeGuide processor to form an accurate real-time image. The mobile receiver dish is adjusted such that the image seen is stable. Images showing the colonoscope configuration are displayed alongside the endoscopy view in a picture-in-picture format that is refreshed several times per second. The safety of the device has been noted in previous studies and does not interfere with implantable cardiac devices.

\section{Study procedures}

Baseline demographics including age, sex, and body mass index (BMI) were recorded in all subjects. All colonoscopies were performed using standard protocol for bowel preparation using split-dose bowel preparations. Written consent was obtained from all patients. Procedures were performed by three experienced endoscopists. All endoscopies were performed using the Olympus 190 series (EVIS EXERA III, CF-HQ190 L, Olympus, America) adult colonoscopies with integrated ScopeGuide technology. Prior to the study, all the endoscopists were familiar with using the assist device. Conscious sedation was administered to all patients and medications administered before the examination and during the examination were dependent on patient discomfort or pain during the procedure.

Sealed, sequentially-numbered envelopes with trial number and allocation group were not opened until written consent was obtained. Randomization was performed immediately before colonoscopy to avoid time for allocation bias to either of the endoscopicsts. After randomization, all patients but neither the endoscopists nor assisting staff were blinded to the insertion method. All patients were then placed in the left lateral decubitus position and underwent conscious sedation and colonoscopy per standard procedure. Conscious sedation with fentanyl and midazolam were titrated according to the endoscopist's practice. If randomization was to ScopeGuide-assisted colonoscopy, prior to insertion of the colonoscopy, the device was switched on to $3 \mathrm{D}$ external image screen, appropriately calibrated, and the ScopeGuide receiver dish position was confirmed. Manual pressure and patient position change were performed typically by an endoscopy nurse or technician at the discretion of the endoscopist. 


\section{Outcomes}

The primary outcome measure was cecal intubation time (CIT) recorded by the nurse assisting with the procedure. This was measured as time from scope insertion to intubation of the cecal tip and documentation of important landmarks such as the appendiceal orifice and/or the ileocecal valve. Time was subtracted for polyp removal during insertion. Secondary outcomes included use of manual pressure, position changes, sedation requirements and patient discomfort levels by visual analogue scale (VAS). These were recorded by an assisting nurse once the procedure was completed. Patient discomfort scores were completed in recovery. Colonoscopy failure was defined as inability to intubate the cecum. Quality of preparation was documented using the Boston Bowel Preparation Scale (BBPS) score.

Secondary outcomes also included provider and assistant assessments of procedural difficulty and severity of endoscope looping. Polyps removed or any other abnormalities detected during the procedure were recorded. Any adverse events (AEs) were also recorded. Patients with incomplete colonoscopies (e.g. due to poor prep) were excluded from primary outcome analysis (CIT), but were included for secondary outcome analyses. Planned a priori subgroup analyses included stratification by age, gender, and BMI. Other outcomes such as mean time in minutes to complete colonoscopy, mean number of mechanical loops required to complete the procedure for each arm and number of mean external maneuvers per procedure between groups was initially planned for evaluation, however, due to impracticality and the relevance of these measures, they were not performed or included in the final analysis.

\section{Statistical analysis}

Standard descriptive statistics were used to assess distribution of study variables. Mean CIT in the intervention and control groups were compared using Wilcoxon test for non-parametric data. Categorical secondary outcomes (e.g. use of manual pressure and patient position change) were compared using chi-square or Fisher's exact tests. Differences were considered statistically significant at an alpha level $<0.05$.

Previous studies have shown that an approximate $20 \%$ difference in procedure time can be achieved by a change in starting position. Mean time to reach the cecum has been reported as 6.4 minutes with standard deviation of 3 minutes. To detect a $20 \%$ difference with $80 \%$ power and $\alpha=0.05$ would require a total sample size of 150 patients in each arm.

\section{Results}

Three hundred patients were randomized to ScopeGuide ( $\mathrm{n}=$ $150)$ vs. standard $(n=150)$. Three patients were excluded as they did not have complete data (2 from the ScopeGuide and 1 from the standard group) ( $\triangleright \mathbf{F i g} . \mathbf{1}$ ). Study groups were similar with regards to age, sex, race, BMI and Charlson comorbidity index ( $\triangleright$ Table 1$)$. Mean age of the patients was $56 \pm 9$ years. Colonoscopy was incomplete in seven patients because of poor preparation. The three endoscopists performing the pro-
Enrollment

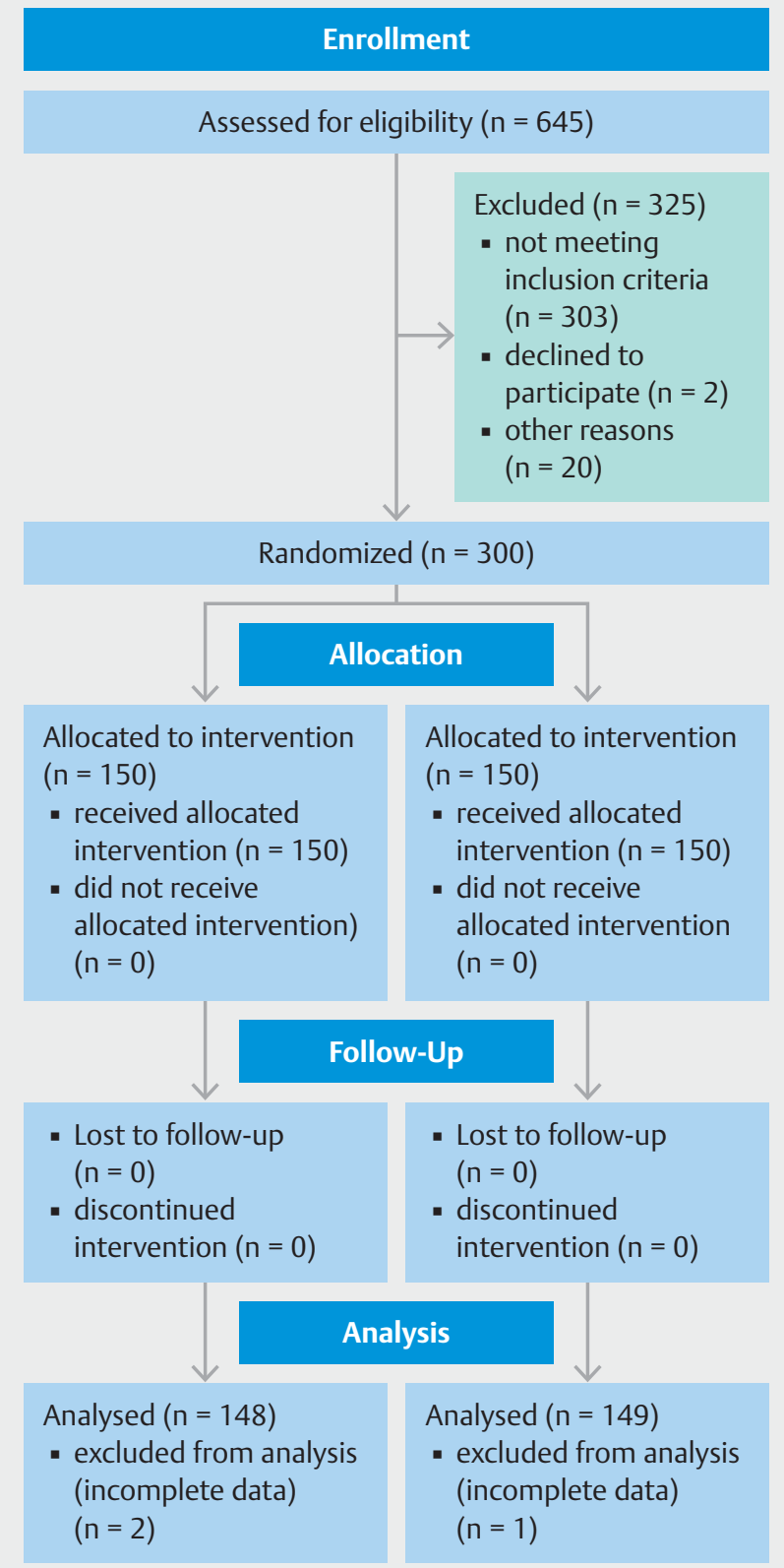

- Fig. 1 Consort algorithm for randomization of patients.

cedure had from 3 to 25 years of experience since fellowship training.

Mean CIT was similar for the ScopeGuide and standard groups ( $4.6 \pm 3$ vs. $4.3 \pm 3.1$ minutes; difference $-0.3 ; 95 \% \mathrm{Cl}$, -0.9 to $0.4 ; P=0.46)(\triangleright$ Table 2$)$. There were no statistically significant differences in frequency of manual pressure applied ( $16.7 \%$ for Scope-guided vs. $19.1 \%$ for standard; $P=0.65$ ) or position changes ( $8.8 \%$ for Scope-guided vs. $11.4 \%$ standard; $P=0.56)$. There was a trend for increased use of stiffener in the standard group compared to Scope guide ( $24.8 \%$ vs. $15 \%$, $P=0.04)$ Patient discomfort scores did not differ between either group (mean VAS: 1.7 for Scope-guided vs. 1.3 for standard; $P=0.09$ ). Sedation requirements showed lesser use 
- Table 1 Baseline characteristics between groups.

\begin{tabular}{|c|c|c|c|}
\hline & $\begin{array}{l}\text { Scope Guide } \\
(N=148)\end{array}$ & $\begin{array}{l}\text { Standard } \\
(\mathrm{N}=149)\end{array}$ & $P$ value \\
\hline Male, n (\%) & $78(52.7)$ & $87(58.4)$ & 0.32 \\
\hline Age (years), mean (SD) & $56(9)$ & $56(8)$ & 0.95 \\
\hline $\begin{array}{l}\text { Race, n (\%) } \\
\text {. White } \\
\text {. Black } \\
\text { - Other }\end{array}$ & $\begin{array}{l}72(48.7) \\
71(48.0) \\
5(3.4)\end{array}$ & $\begin{array}{l}73(49.0) \\
72(48.3) \\
4(2.7)\end{array}$ & 0.94 \\
\hline Body mass index, mean (SD) & $31.7(7.4)$ & $31.9(5.7)$ & 0.81 \\
\hline Charleson Comorbidity Index, mean (SD) & $2.1(1.6)$ & $1.9(1.4)$ & 0.29 \\
\hline $\begin{array}{l}\text { Endoscopist experience, n (\%) } \\
\text { - Professor } \\
\text { - Associate } \\
\text { - Assistant }\end{array}$ & $\begin{array}{l}34(23.0) \\
80(54.1) \\
34(23.0)\end{array}$ & $\begin{array}{l}51(34.2) \\
58(39.0) \\
40(26.9)\end{array}$ & 0.02 \\
\hline Boston bowel preparation score (BBPS), mean (SD) & $7.4(1.8)$ & $7.7(1.6)$ & 0.10 \\
\hline History of abdominal surgery, $\mathrm{n}(\%)$ & $47(31.8)$ & $44(29.5)$ & 0.71 \\
\hline $\begin{array}{l}\text { Medication use, n (\%) } \\
\text { - Anxiolytics } \\
\text { - Narcotics } \\
\text { - Antidepressants }\end{array}$ & $\begin{array}{l}12(8.2) \\
15(10.2) \\
31(21.1)\end{array}$ & $\begin{array}{l}19(12.8) \\
19(12.8) \\
32(21.5)\end{array}$ & $\begin{array}{l}0.25 \\
0.59 \\
1.00\end{array}$ \\
\hline Adenoma removal, $\mathrm{n}(\%)$ & $39(27.1)$ & $42(28.2)$ & 0.90 \\
\hline
\end{tabular}

- Table 2 Primary outcome measures between two groups.

\begin{tabular}{|c|c|c|c|}
\hline & $\begin{array}{l}\text { ScopeGuide } \\
(\mathrm{N}=148)\end{array}$ & $\begin{array}{l}\text { Standard } \\
(\mathrm{N}=149)\end{array}$ & $P$ value \\
\hline $\mathrm{CIT}$, mean (SD) & $4.6(3.0)$ & $4.3(3.1)$ & 0.46 \\
\hline $\begin{array}{l}\text { Completion, n (\%) } \\
\text { - Failure } \\
\text { - Complete }\end{array}$ & $\begin{array}{l}5(3.4) \\
143(96.6)\end{array}$ & $\begin{array}{l}2(1.3) \\
147(98.7)\end{array}$ & 0.28 \\
\hline Application of pressure, $\mathrm{n}(\%)$ & $24(16.9)$ & $28(19.1)$ & 0.65 \\
\hline Need for position change, $n$ (\%) & $17(11.4)$ & $13(8.8)$ & 0.56 \\
\hline Use of stiffener, n (\%) & $22(15.0)$ & $37(24.8)$ & 0.04 \\
\hline Complication, n (\%) & $0(0.0)$ & $0(0.0)$ & - \\
\hline
\end{tabular}

of midazolam ( $3.9 \mathrm{mg}$ vs. $4.7 \mathrm{mg}, P=0.003$ ) in the Scope-guided group, while we observed a statistically nonsignificant $(P=0.09)$ difference of $6.8 \mathrm{mg}$ in use of fentanyl between the Scope-guided vs. standard groups, respectively $(62.1 \mathrm{mg}$ vs. $68.9 \mathrm{mg})$. No major AEs were observed in either group $(P=1.00)$. There were five patients in the ScopeGuide group $(2$ =discomfort, 1 = severe diverticulosis, 1 = redundant colon, 1 = unreducible inguinal hernia) and 2 ( 1 = severe diverticulosis, 1 =acute angulation with looping) in the standard group who had failure of cecal intubation.
Secondary outcomes with regards to operator difficulty showed similarity between the ScopeGuide and the standard groups (mean VAS: 3.1 vs. $2.9 ; P=0.45$ ), respectively. The assistant also recorded similar subjectively perceived difficulty between groups (mean VAS: 1.5 vs. $1.7 ; P=0.18$ ) ( $>$ Table 3 ).

Subgroup analysis based on gender, BMI $>30$ and age $>60$, showed no difference in CIT, use of manual pressure or change in position ( $\vee$ Table 4$)$. 
- Table 3 Secondary outcome measures between two groups.

\begin{tabular}{|c|c|c|c|}
\hline & $\begin{array}{l}\text { ScopeGuide } \\
(N=148)\end{array}$ & $\begin{array}{l}\text { Standard } \\
(\mathrm{N}=149)\end{array}$ & $P$ value \\
\hline Operator difficulty (VAS), mean (SD) & $3.1(2.0)$ & $2.9(1.7)$ & 0.45 \\
\hline Operator assistant difficulty (VAS), mean (SD) & $1.5(1.7)$ & $1.7(1.7)$ & 0.38 \\
\hline Patient-reported difficulty (VAS), mean (SD) & $1.7(2.2)$ & $1.3(2.0)$ & 0.18 \\
\hline $\begin{array}{l}\text { Sedation (in mg), mean (SD) } \\
\text { - Versed } \\
\text { " Fentanyl } \\
\text { - Demerol }\end{array}$ & $\begin{array}{l}3.9(2.2) \\
62.1(27.2) \\
43.7(13.4)\end{array}$ & $\begin{array}{l}4.7(2.6) \\
68.9(28.2) \\
46.2(13.5)\end{array}$ & $\begin{array}{l}0.003 \\
0.09 \\
0.35\end{array}$ \\
\hline
\end{tabular}

VAS, visual analog scale; SD, standard deviation

- Table 4 Stratified outcome measures between two groups.

\begin{tabular}{|c|c|c|c|}
\hline Stratified outcome & Standard & Scope guide & $P$ value \\
\hline \multicolumn{4}{|l|}{ Sex stratification } \\
\hline $\begin{array}{l}\text { Female } \\
\text { - CIT (mean/SD) } \\
\text { - Use of pressure (yes) \% } \\
\text { - Change in position(yes) \% }\end{array}$ & $\begin{array}{l}5.31(3.26) \\
13(21.3) \\
7(11.3)\end{array}$ & $\begin{array}{l}5.29(3.52) \\
13(19.1) \\
8(11.4)\end{array}$ & $\begin{array}{l}0.97 \\
0.83 \\
1.00\end{array}$ \\
\hline $\begin{array}{l}\text { Male } \\
\text { - CIT (mean/SD) } \\
\text { - Use of pressure (yes) \% } \\
\text { - Change in position (yes)\% }\end{array}$ & $\begin{array}{l}3.61(2.74) \\
15(17.4) \\
10(11.49)\end{array}$ & $\begin{array}{l}3.92(2.17) \\
11(14.9) \\
5(6.5)\end{array}$ & $\begin{array}{l}0.44 \\
0.67 \\
0.29\end{array}$ \\
\hline \multicolumn{4}{|l|}{ Age stratification } \\
\hline $\begin{array}{l}\text { Age }<60 \\
\text { - CIT (mean/SD) } \\
\text { - Use of pressure (yes) \% } \\
\text { - Change in position (yes) \% }\end{array}$ & $\begin{array}{l}3.89(2.47) \\
15(15.5) \\
9(9.2)\end{array}$ & $\begin{array}{l}3.80(1.91) \\
12(13.0) \\
4(4.2)\end{array}$ & $\begin{array}{l}0.78 \\
0.68 \\
0.25\end{array}$ \\
\hline $\begin{array}{l}\text { Age > } 60 \\
\text { - CIT (mean/SD) } \\
\text { - Use of pressure (yes) \% } \\
\text { - Change in position (yes) \% }\end{array}$ & $\begin{array}{l}5.12(3.90) \\
13(26.0) \\
8(15.7)\end{array}$ & $\begin{array}{l}6.02(3.93) \\
12(24.0) \\
9(17.7)\end{array}$ & $\begin{array}{l}0.26 \\
1.0000 \\
1.0000\end{array}$ \\
\hline \multicolumn{4}{|l|}{ BMI stratification } \\
\hline $\begin{array}{l}<30 \\
\text { " CIT (mean/SD) } \\
\text { " Use of pressure (yes) \% } \\
\text { " Change in position (yes) \% }\end{array}$ & $\begin{array}{l}4.79(3.81) \\
12(20.3) \\
8(13.6)\end{array}$ & $\begin{array}{l}4.74(3.18) \\
10(13.9) \\
3(4.1)\end{array}$ & $\begin{array}{l}0.94 \\
0.36 \\
0.06\end{array}$ \\
\hline $\begin{array}{l}>30 \\
\text { " CIT (mean/SD) } \\
\text { " Use of pressure (yes) \% } \\
\text { " Change in position (yes) \% }\end{array}$ & $\begin{array}{l}4.10(2.54) \\
15(19.5) \\
9(11.4)\end{array}$ & $\begin{array}{l}4.36(2.72) \\
13(18.8) \\
9(12.5)\end{array}$ & $\begin{array}{l}0.56 \\
1.00 \\
1.00\end{array}$ \\
\hline
\end{tabular}

\section{Discussion}

We performed a prospective randomized controlled trial evaluating use of the ScopeGuide-assisted device in enabling better outcomes for colonoscopy. We found that the overall usefulness was limited in our practice. There were no differences in patient satisfaction or difficulty of the procedure. The current $3 \mathrm{D}$ technology incorporates real-time visualization of the colo- noscope as it advances through the colon ( $\triangleright$ Fig. 2 ). This especially is noteworthy as it enables the operator to reduce loop formation especially in areas of the sigmoid colon with subsequent straightening of the scope. However, in our study, this enhanced feature did not alter primary or secondary outcomes such as CIT, patient discomfort scores or use of ancillary maneuvers. There was a trend in more use of midazolam and use of stiffener in the standard group though this did not concur with 


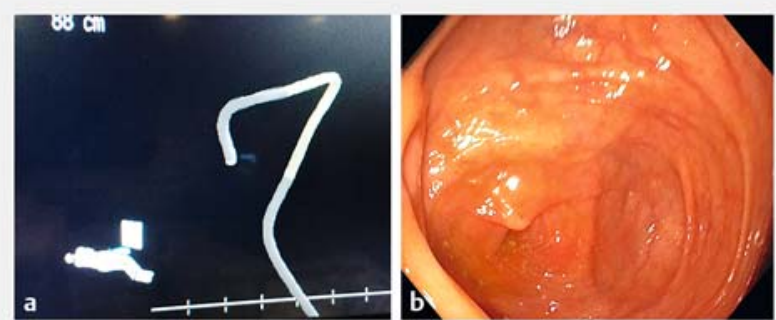

- Fig. 2 a ScopeGuide imaging in completely reduced position. b Accompanying cecal image,

the rest of the results. Our findings may have explanations related to the fact that for experienced endoscopists who are primarily trained traditionally, application of navigation-aiding tools such the ScopeGuide did not make a significant difference in their skills as arguably, maneuverability of the endoscope in their hands is dependent on factors such as manual feel, appropriate torque engagement, and adequate endoscopic visualization for appropriate reduction while advancing the colonoscope. Besides visual orientation cues, several motor skills (independent cecal intubation, total and withdrawal time, loop reduction) and cerebromotor skills (appropriate mucosal evaluation, preparation evaluation, pathology recognition, appropriate tool selection and sedation) are important combining metrics that indicate an efficient colonoscopy procedure. Technical challenges during colonoscopy are expected in patients who had previous abdominal surgery, diverticular disease, or female gender; however, subgroup analysis of these factors did not change the measured procedural outcomes. Training on virtual simulators has been shown to have benefit in nurturing endoscopy skills in the long term; however, our study reflected an actual performance in a routine clinical setting beyond the training room [5].

Several studies have demonstrated improved outcomes with use of ScopeGuide [6]. The meta-analysis by Mark-Christensen et al looked at 13 randomized trials and demonstrated that magnetic endoscopic imaging (MEI) was significantly associated with lower risk of failed cecal intubation, shorter cecal intubation time (mean difference of 0.58 minutes, $95 \% \mathrm{Cl} 0.28-0.88$; $P<0.001$ ) and lower pain scores estimated by VAS (mean difference $0.45 \mathrm{~cm}, 95 \% \mathrm{Cl} 0.03-0.86 ; P=0.03$ ) compared with conventional colonoscopy [4]. Subgroup analysis conferring to endoscopist experience showed this factor had no influence on failure; however reduced CIT was noted in the more experienced group, which was different from our study. Notably, in this analysis, none of the studies included were adequately powered to detect differences in complication rates, as well as the uncertainness of the cecal intubation where only three of 13 studies used a definition to ensure that the cecum was reached. There were also divergent definitions of endoscopist experience whereas in our study each endoscopist had performed a minimum of $>1000$ unassisted procedures. Nevertheless, considering the between-study discrepancies and heterogeneity that could have led to uncertainty in the meta-analysis estimates, the discrepancies were most likely balanced within the studies and therefore did not introduce bias.

Looping of the endoscope and negotiating across sharp bends are challenging situations during colonoscopy and can reflect on body stature. Looping can occur in up to $90 \%$ of colonoscopies and is the major cause of patient discomfort as well as increased procedure time [7-11]. Both lean and obese patients can be independent limiting factors for a smooth endoscopy [11]. Loop formation can be more pronounced in slender patients with acute angles while position changes and ancillary pressure may be increasingly required in obese patients. Stratifying by BMI nonetheless did not show an advantage of the device in our study. The study is also similar in outcomes to a previous study by Shergill et al although the latter used MEI performance in a predominantly unsedated veteran-based population with almost all male patients [12]. Stratifying by sex or age did not change the outcomes in our study. Moreover, they used an older version of the ScopeGuide (MEl colonoscope (CF-Q140DL, Olympus America) compared to the newer integrated technology that we used in our study (Olympus 190 series (EVIS EXERA III, CF-HQ190 L, Olympus, America).

Our study is unique in that the strengths lie in its randomized design as well as in the generalizability of the patient population including those seen in a routine ambulatory clinical endoscopic practice. Our center involved a high-volume academic center with endoscopists familiar to open-access system of endoscopy. It involved patients who were given "conscious sedation" using midazolam and fentanyl as compared to a growing trend of performing these procedures under deep sedation using propofol administered by anesthesia professionals [13]. Despite moderate sedation, the outcomes were within the scope of a traditional practice with fewer side effects and reasonable patient satisfaction scores achieving good standard-of-care quality measures, which is in comparison to another study that showed lower doses of propofol in the MEl group [14]. Use of deep sedation can limit position changes. It remains to be seen if this modality can be effective in community practice centers as compared to academic areas. It may also be pointed out that the study used the latest version of the assist guide technology (Olympus 190 series) compared to previous studies which used earlier versions. The newer series incorporates RIT which is standard on EVIS EXERA III 190 Series colonoscopies and features a unique combination of three proprietary technologies: PB (Passive Bending), HFT (High Force Transmission), and Variable Stiffness. These technologies are meant to work together to improve ease of insertion and operator control, which may help to minimize patient discomfort and thereby enhance procedural efficiency [15]. However, despite improved design, our study failed to find a major difference in outcomes. Given these results, it might also be worth noting the cost of investing in this technology: MEI ScopeGuide, model number UPD-3, Olympus America: $\$ 21,300$ [16].

Our study has some noteworthy limitations, as it was performed in a single center and reflects the practice patterns of this area and investigator expertise. We could not blind the operator to subject randomization as it is not feasible to perform such a study and that could have introduced investigator 
bias. There were patients in the practice who had previously described difficult colonoscopies and therefore their procedures were performed using a smaller-diameter pediatric colonoscope. These "difficult" patients were not included and therefore were a limitation to the study with regard to whether the assist device was useful in this subset of patients. At the time of this study, the pediatric colonoscopes were not equipped for the ScopeGuide and therefore the study could not be extended to those patients. Moreover, $50 \%$ of those patients screened were not included in the final randomization, which therefore impacts the overall results and generalizability, even though this seems to reflect clinical practice. We did, however, record the number of patients in whom a switch was made to another scope if the initial maneuver failed. Our study was also limited to experienced endoscopists and therefore could not assess the potential of the device in trainee performance and could not elucidate a potential impact of ScopeGuide on the endoscopy learning curve.

\section{Conclusion}

In conclusion, while the ScopeGuide may be a useful tool, based on our results, it does not seem to affect the performance of experienced endoscopists in a "real-world" clinical endoscopy practice center. Given outcomes of previous studies, it may have value during training endoscopists and be vital stepping stones to advance their skill sets. Use of this technology will have to be tested in further studies, particularly in patients who have undergone failed or had incomplete colonoscopies due to various reasons such as adhesive disease, tortuous and reductant colons, to specifically examine these challenging situations. In such patients, in addition to performing maneuvers, the adoption of this technology while switching to pediatric colonoscopies or single- balloon enteroscopies for completion will need to be further studied.

\section{Competing interests}

None

\section{References}

[1] Seeff LC, Richards TB, Shapiro JA et al. How many endoscopies are performed for colorectal cancer screening? Results from CDC's survey of endoscopic capacity Gastroenterology 2004; 127: 1670 - 1677
[2] Kaltenbach T, Leung C, Wu K et al. Use of the colonoscope training model with the colonoscope 3D imaging probe improved trainee colonoscopy performance: a pilot study. Dig Dis Sci 2011; 56: 1496 1502

[3] Wehrmann K, Frühmorgen P. Evaluation of a new three-dimensional magnetic imaging system for use during colonoscopy. Endoscopy 2002; 34: 905-908

[4] Mark-Christensen A, Brandsborg S, Iversen LH. Magnetic endoscopic imaging as an adjuvant to elective colonoscopy: a systematic review and meta-analysis of randomized controlled trials. Endoscopy 2015; 47: $251-261$

[5] Singh S, Sedlack RE, Cook DA. Effects of simulation-based training in gastrointestinal endoscopy: a systematic review and meta-analysis. Clin Gastroenterol Hepatol 2014; 12: 1611 - 1623 e1614

[6] Cheung HY, Chung CC, Kwok SY et al. Improvement in colonoscopy performance with adjunctive magnetic endoscope imaging: a randomized controlled trial. Endoscopy 2006; 38: 214-217

[7] Rex DK, Goodwine BW. Method of colonoscopy in 42 consecutive patients presenting after prior incomplete colonoscopy. Am J Gastroenterol 2002; 97: 1148-1151

[8] Shah SG, Brooker JC, Williams CB et al. Effect of magnetic endoscope imaging on colonoscopy performance: a randomised controlled trial. Lancet 2000; 356: $1718-1722$

[9] Shah SG, Brooker JC, Thapar C et al. Patient pain during colonoscopy: an analysis using real-time magnetic endoscope imaging. Endoscopy 2002; 34: $435-440$

[10] Cheng WB, Moser MA, Kanagaratnam S et al. Analysis of and mathematical model insight into loop formation in colonoscopy. Proceedings of the Institution of Mechanical Engineers Part H. J Engin Med 2012; 226: $858-867$

[11] Witte TN, Enns R. The difficult colonoscopy. Can J Gastroenterol 2007; 21: $487-490$

[12] Shergill AK, McQuaid KR, Deleon A et al. Randomized trial of standard versus magnetic endoscope imaging colonoscopes for unsedated colonoscopy. Gastrointest Endosc 2012; 75: 1031 - 1036 e1031

[13] Vargo JJ, Niklewski PJ, Williams JL et al. Patient safety during sedation by anesthesia professionals during routine upper endoscopy and colonoscopy: an analysis of 1.38 million procedures. Gastrointest Endosc 2017; 85: $101-108$

[14] Klare P, Hartrampf B, Haller B et al. Magnetic endoscope imaging for routine colonoscopy: impact on propofol dosage and patient safety a randomized trial. Endoscopy 2016; 48: 916 - 922

[15] Pasternak A, Szura M, Solecki R et al. Impact of responsive insertion technology (RIT) on reducing discomfort during colonoscopy: randomized clinical trial. Surg Endosc 2017; 31: 2247-2254

[16] Trindade AJ, Lichtenstein DR, Aslanian HR et al. Devices and methods to improve colonoscopy completion (with videos). Gastrointest Endosc 2018; 87: 625-634 